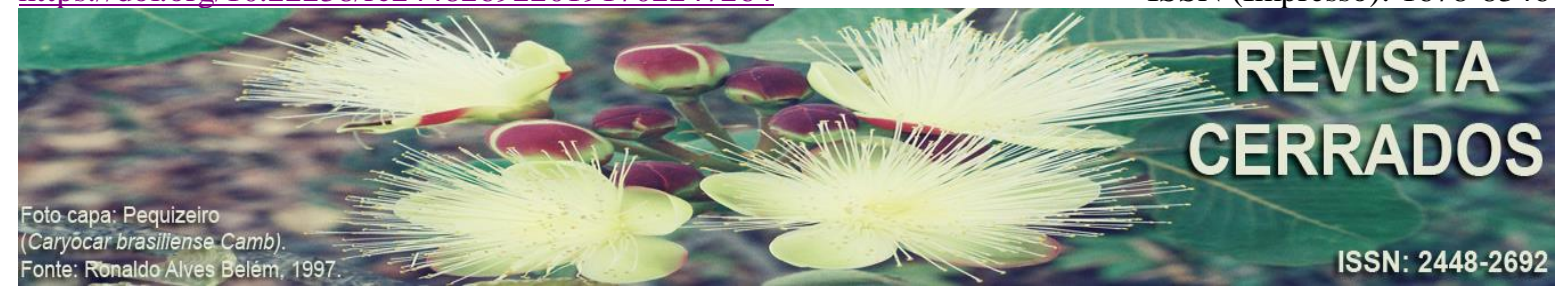

\title{
GERAÇÃO DE MODELO DIGITAL DO TERRENO E EXTRAÇÃO DE PARÂMETROS GEOMORFOMÉTRICOS A PARTIR DE DADOS COLETADOS POR AERONAVES REMOTAMENTE PILOTADAS
}

\author{
GENERATION OF DIGITAL TERRAIN MODEL AND EXTRACTION \\ OF GEOMORPHOMETRIC PARAMETERS FROM DATA \\ COLLECTED BY REMOTELY PILOT AIRCRAFT
}

\section{GENERACIÓN DE MODELO DIGITAL DEL TERRENO Y EXTRACCIÓN DE PARÁMETROS GEOMORFOMÉTRICOS DE LOS DATOS RECOPILADOS POR LA AERONAVE PILOTO REMOTO}

\author{
Otacílio Lopes de Souza da Paz \\ Universidade Federal do Paraná - UFPR \\ E-mail: <otacilio.paz@gmail.com>.

\section{Tony Vinicius Moreira Sampaio \\ Universidade Federal do Paraná - UFPR E-mail: < tonysampaio@ufpr.br>}

\begin{abstract}
RESUMO
A morfometria do relevo pode ser analisada pela extração de parâmetros geomorfométricos a partir de modelos digitais do terreno (MDT). MDT de alta resolução espacial pode ser obtido por aerolevantamento com aeronave remotamente pilotada (RPA). Objetiva-se gerar MDT a partir de aerolevantamento com RPA e analisar os parâmetros geomorfométricos extraídos. Foi realizado aerolevantamento com uma RPA modelo Phantom 4 Advanced em uma bacia de primeira ordem localizada a $3 \mathrm{~km}$ da cidade de Medianeira (oeste do Paraná). Os dados coletados foram processados no Agisoft PhotoScan 1.2.4. A nuvem de pontos gerada passou por filtragens e foi interpolada via krigagem no ArcGIS 10.1, resultando no MDT. Os parâmetros geomorfométricos foram extraídos no ArcGIS 10.1. A área de estudo apresenta em maior parte vertentes planar-retilíneas com declividade de até 5 graus e baixos valores de ITU. No vale do rio de primeira ordem, foram encontradas vertentes convergentes-côncavas marcadas por declividades de 5 a 9 graus, associado a altos valores de ITU. O MDT gerado permitiu a identificação de feições de detalhe observadas em campo. Conclui-se que dados coletados por RPA tem grande potencial para caracterização morfométrica em detalhe do relevo.
\end{abstract}


PAZ, O. L. S.; SAMPAIO, T. V. M.

Geração de modelo digital do terreno e extração de parâmetros geomorfométricos a partir de dados coletados por aeronaves remotamente pilotadas

Palavras-chave: Geomorfologia; Geomorfometria; Análise Digital do Relevo; Atributos topográficos.

\begin{abstract}
The relief morphometry can be analyzed by the extraction of geomorphometric parameters from digital terrain models (MDT). High resolution MDT can be obtained by aero-surveys with remotely piloted aircraft (RPA). The objective is to generate MDT from aerial surveying with RPA and to analyze the extracted geomorphometric parameters. It was performed aerial survey with a RPA model Phantom 4 Advanced in a first order basin located $3 \mathrm{~km}$ from the city of Medianeira (western Paraná). The data collected was processed in Agisoft PhotoScan 1.2.4. The generated point cloud passed through filtering and was interpolated via kriging in ArcGIS 10.1, resulting in the MDT. The geomorphometric parameters were extracted in SAGA GIS 2.3.2. The study area presents mostly planar-rectilinear slopes with slopes of up to 5 degrees and low values of UTI. In the first-order river valley, convergent-concave slopes were found marked by slopes of 5 to 9 degrees, associated with high UTI values. The generated MDT allowed the identification of features observed in the field. In conclusion, that data collected by RPA has great potential for morphometric characterization in relief detail.
\end{abstract}

Keywords: Geomorphology; Geomorphometry; Digital Relay Analysis; Topographical attributes.

\title{
RESUMEN
}

Se puede analizar la morfometría en relieve extrayendo parámetros geomorfométricos de los modelos digitales del terreno (MDT). El MDT de alta resolución espacial puede obtenerse mediante reconocimiento aéreo de aeronaves pilotadas a distancia (RPA). El objetivo es generar MDT a partir del levantamiento aéreo RPA y analizar los parámetros geomorfométricos extraídos. Se encuestó un modelo RPA Phantom 4 Advanced en una cuenca de primer orden ubicada a $3 \mathrm{~km}$ de la ciudad de Medianeira (oeste de Paraná). Los datos recopilados se procesaron en Agisoft PhotoScan 1.2.4. La nube de puntos generada se filtró y se interpoló a través de kriging en ArcGIS 10.1, lo que resultó en MDT. Los parámetros geomorfométricos se extrajeron en ArcGIS 10.1. El área de estudio tiene en su mayoría pendientes planas rectilíneas de hasta 5 grados y bajos valores de ITU. En el valle del río de primer orden, se encontraron pendientes cóncavas convergentes marcadas por pendientes de 5 a 9 grados, asociadas con altos valores de IU. Se concluye que los datos recopilados por RPA tienen un gran potencial para la caracterización morfométrica en detalle en relieve.

Palabras-clave: Geomorfología; Geomorfometría; Análisis digital de socorro; Atributos topográficos. 
PAZ, O. L. S.; SAMPAIO, T. V. M.

Geração de modelo digital do terreno e extração de parâmetros geomorfométricos a partir de dados coletados por aeronaves remotamente pilotadas

\section{INTRODUÇÃO}

A análise morfométrica do relevo pode ser feita por meio de técnicas de sistema de informações geográficas (SIG) em modelos digitais do terreno. Os modelos digitais do terreno (MDT) podem ser definidos como representações em formato digital da topografia da terra (RAAFLAUB; COLLINS, 2006).

Dentro da análise quantitativa do relevo, a geomorfometria se dedica a quantificar e parametrizar a superfície terrestre (PIKE, 2000; SILVA; OKA-FIORI; SILVEIRA, 2017). A extração de informações quantitativas do relevo (parâmetros geomorfométricos) a partir de MDT subsidiam análises dentro das ciências da terra (SILVA; OKA-FIORI; SILVEIRA, 2017).

Parâmetros geomorfométricos podem ser definidos como medidas ou índices do relevo, sendo divididos em parâmetros locais e parâmetros regionais (OLAYA, 2009). Os parâmetros locais se pautam em propriedades geométricas ou estatísticas do terreno, envolvendo análise de vizinhança (janelas amostrais fixa ou móveis) com operações matemáticas como média, mediana ou amplitude (OLAYA, 2009; SILVA; OKA-FIORI; SILVEIRA, 2017). Já os parâmetros regionais podem se referir a propriedades hidrológicas do terreno, considerando a conectividade entre os pixels (OLAYA, 2009).

A extração de parâmetros geomorfométricos pode ser aplicada em estudos de fragilidade ambiental (MARION; MELLO FILHO; SILVA, 2010), mapeamentos de solos (ARRUDA; DEMATTÊ; SILVA CHAGAS, 2013), mapeamentos geomorfológicos (SILVEIRA et al., 2012), desastres naturais (SAHA; GUPTA; ARORA, 2002), entre outros.

Os MDT podem ser gerados pela interpolação de dados altimétricos obtidos por métodos geodésicos ou topográficos utilizando algoritmos como Inverse Distance Weighting (IDW) (ARUN, 2013), krigagem (REICHEL; SAMPAIO, 2018) ou ANUDEM (SILVEIRA; SILVEIRA, 2016). MDT também podem ser gerados por dados coletados por sensores passivos e ativos (TACHIKAWA et al., 2011; SOPCHAKI; SAMPAIO, 2016; SILVA; OKAFIORI; SILVEIRA, 2017).

MDT de alta resolução espacial podem ser obtidos a partir de aerolevantamentos com Aeronaves Remotamente Pilotadas (RPA, do inglês Remotely Piloted Aircraft). Os aerolevantamentos com RPA podem gerar MDT e ortomosaico. As RPA integram dados 
PAZ, O. L. S.; SAMPAIO, T. V. M.

Geração de modelo digital do terreno e extração de parâmetros geomorfométricos a partir de dados coletados por aeronaves remotamente pilotadas

inerciais e dados Global Navigation Satellite System (GNSS) para navegação e essas informações são gravadas nos metadados das imagens captadas (GUJJAR et al., 2017; MOHAMMAD et al., 2017).

São utilizados nos processamentos desses dados algoritmos de "estrutura a partir de movimento" (do inglês, Structure-from-Motion - SfM), que permitem a reconstrução tridimensional da área de estudo em alta resolução espacial (centímetros), gerando nuvens de pontos (WESTOBY et al., 2012). A nuvem de pontos contém o sistema de coordenadas X e Y (latitude e longitude) e Z (altitude).

Trabalhos já abordaram a qualidade vertical dos MDT e posicional dos ortomosaicos gerados por aerolevantamentos com RPA (HUNG et al., 2018; SOPCHAKI et al., 2018). Estes trabalhos constataram que ambos os produtos se enquadram na classe A 1:5.000 do Padrão de Exatidão Cartográfica dos Produtos Cartográficos Digitais (PEC-PCD) (DSG, 2016).

No entanto, estudos que discutam a aplicação dos MDT gerados por aerolevantamentos com RPA para extração de parâmetros geomorfométricos são insipientes. Assim, objetiva-se gerar MDT a partir de aerolevantamento com RPA e analisar os parâmetros geomorfométricos extraídos a partir deste. A seguir é apresentado os procedimentos metodológicos seguidos para atingir tal objetivo.

\section{PROCEDIMENTOS METODOLÓGICOS}

A área de estudo selecionada foi uma bacia hidrográfica de primeira ordem localizada a oeste da cidade de Medianeira (Paraná) (FIGURA 1). O acesso a área de estudo se dá pela BR 277. A área possui 29 hectares e perímetro de $2168,39 \mathrm{~m}$. O rio de primeira ordem pertence a porção montante da bacia do córrego Tigre. 
PAZ, O. L. S.; SAMPAIO, T. V. M.

Geração de modelo digital do terreno e extração de parâmetros geomorfométricos a partir de dados coletados por aeronaves remotamente pilotadas

Figura 1: Localização da área de estudo.

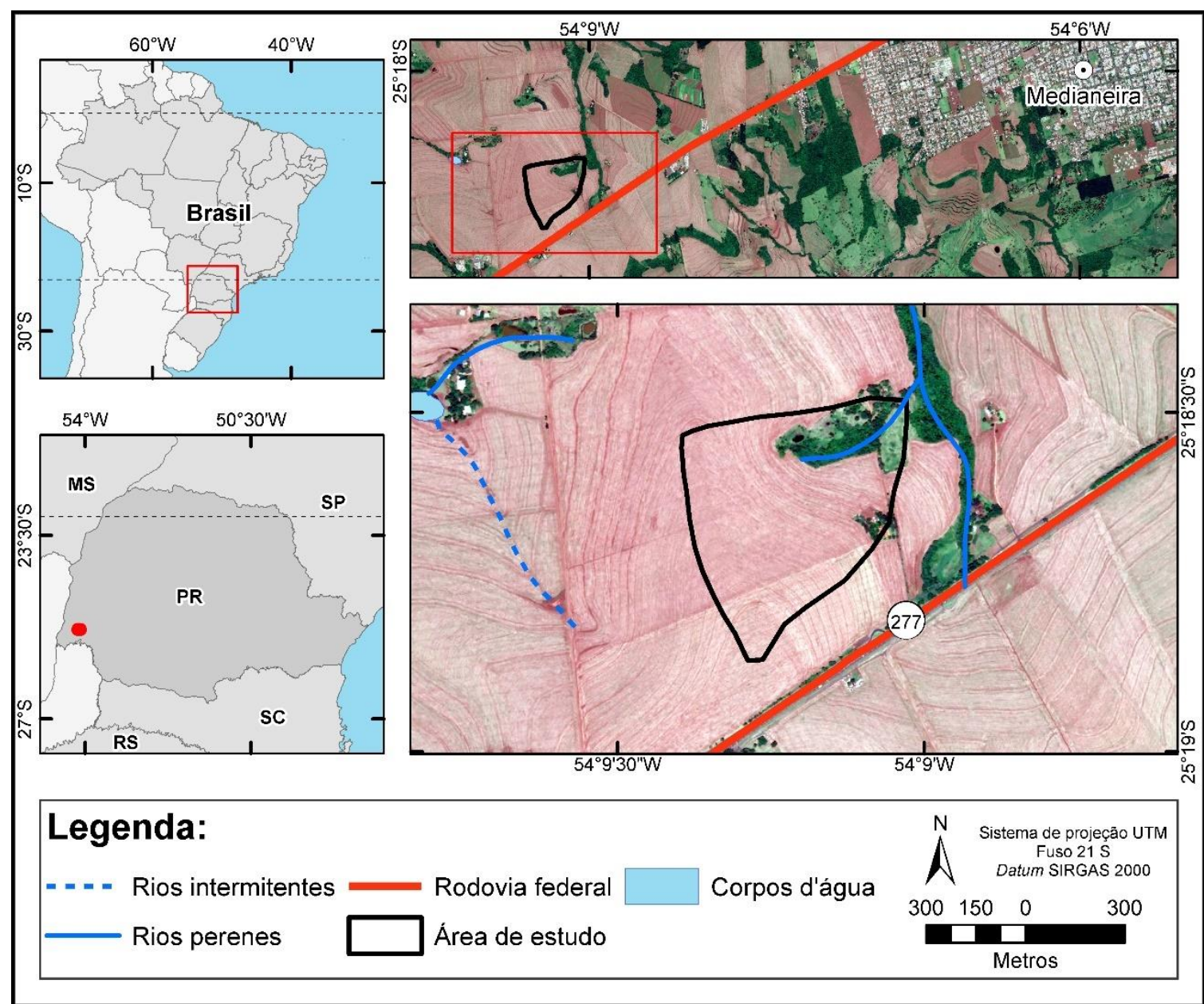

FONTE: Os autores (2019).

Para o aerolevantamento foi utilizado uma RPA modelo Phantom 4 Advanced, produzido pela empresa chinesa $D J I$. O equipamento foi cedido pelo Laboratório de Análise de Padrões Espaciais e Cartografia Temática, vinculado ao Departamento de Geografia da Universidade Federal do Paraná. Em conjunto com a RPA, foi utilizado a controladora e um smartphone para o planejamento de voo.

O planejamento de voo foi realizado no aplicativo Pix4D Capture (Erro! Fonte de referência não encontrada.), delimitado a partir de um Keyhole Markup Language (KML) da bacia de primeira ordem, delimitada pela folha topográfica São Miguel do Iguaçu (MI 2832-4-NO - 1:25.000) acrescido de um buffer de 15 metros para evitar o efeito de borda no MDT. O voo foi definido em 150 metros de altura e o recobrimento lateral (overlap) de $70 \%$. 
PAZ, O. L. S.; SAMPAIO, T. V. M.

Geração de modelo digital do terreno e extração de parâmetros geomorfométricos a partir de dados coletados por aeronaves remotamente pilotadas

Figura 2: Planejamento do aerolevantamento na área de estudo pelo aplicativo Pix4D.

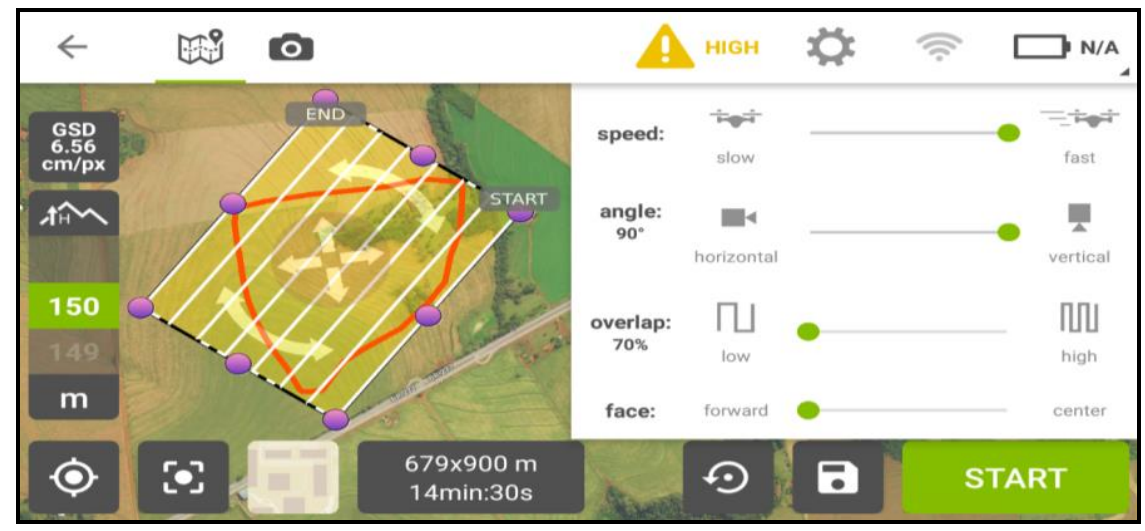

FONTE: Os autores (2019).

O processamento dos dados coletados pela RPA foi realizado no software Agisoft PhotoScan 1.2.4, versão de teste. Não foram utilizados pontos de controle para processamento dos dados. O primeiro passo foi o alinhamento das imagens coletadas pela ferramenta Aligh Photos com Accuracy média (médium). Após, foi realizada a densificação da nuvem de pontos pela ferramenta Build Dense Cloud, com qualidade (quality) média (médium).

A nuvem de pontos se refere a pontos homólogos entre as imagens coletadas com informações em X, Y e Z (latitude, longitude e altitude). Além de representar o terreno, os pontos podem se referir a elementos como árvores, casas, carros ou torres de energia. Para gerar o MDT são interpolados apenas os pontos referentes ao solo, sendo necessário filtrar a nuvem de pontos gerada. Foi utilizada a ferramenta Classify Ground Points do Agisoft PhotoScan 1.2.4 com a seguinte configuração: ângulo máximo de 10 graus, distância máxima de 0.5 metros e tamanho da célula em 1 metro. Após a filtragem ainda pode-se observar pontos referentes a elementos sobre o terreno, sendo estes removidos de forma manual posteriormente.

A partir da nuvem de pontos filtrada foi gerado o modelo digital de superfície MDS (pontos de terreno + outros elementos sob o terreno) pela ferramenta Build DEM. Com o MDS foi gerado o ortomosaico pela ferramenta Build Orthomosaic, exportado com 0,5 metros de resolução espacial. A nuvem de pontos filtrada foi exportada no formato LAS. O arquivo LAS foi carregado no Global Mapper 18.0 e exportado para o formato shapefile (SHP). No ArcGIS 10.1, a nuvem de pontos foi convertida para a projeção UTM - fuso 21 sul - SIRGAS 2000. 
PAZ, O. L. S.; SAMPAIO, T. V. M.

Geração de modelo digital do terreno e extração de parâmetros geomorfométricos a partir de dados coletados por aeronaves remotamente pilotadas

Foram separados 30 mil pontos da nuvem de pontos no ArcGIS 10.1, pela ferramenta Subset Features. Neste momento os pontos foram filtrados manualmente analisando o ortomosaico, visando retirar pontos sobre casas, vegetação ou plantações. Após, foi analisado se os dados apresentam distribuição normal pela ferramenta Histogram do pacote geoestatístico do ArcGIS 10.1, requisito para interpolação dos dados. Foi considerado que a nuvem de pontos apresentava distribuição normal ao se observar o formato de um sino no histograma dos dados e pelo fato de que a média e a mediana estavam com valores próximos.

Antes da interpolação da nuvem de ponto foi analisado os gráficos boxplot e os polígonos de Voronoi visando identificar outliers globais e locais. Para interpolar a nuvem de pontos foi utilizada a krigagem simples. Não foi aplicado remoção de tendência e nem transformação de dados. O tamanho do passo (lag) foi definido com base na distância média entre os pontos, consultada pela ferramenta Average Nearest Neighbor do ArcGIS 10.1. O número de passos foi definido dividindo a maior extensão da bacia pelo tamanho do passo. $\mathrm{O}$ modelo geoestatístico utilizado foi o Gaussiano, sendo o alcance (major range) e o partial sill calibrados até que o modelo apresentasse melhor "encaixe" no semivariograma (FIGURA 3). O MDT gerado foi exportado com resolução espacial de 1 metro e será chamado ao longo do texto de MDT RPA.

Figura 3: Aplicação da krigagem simples na nuvem de pontos gerada.

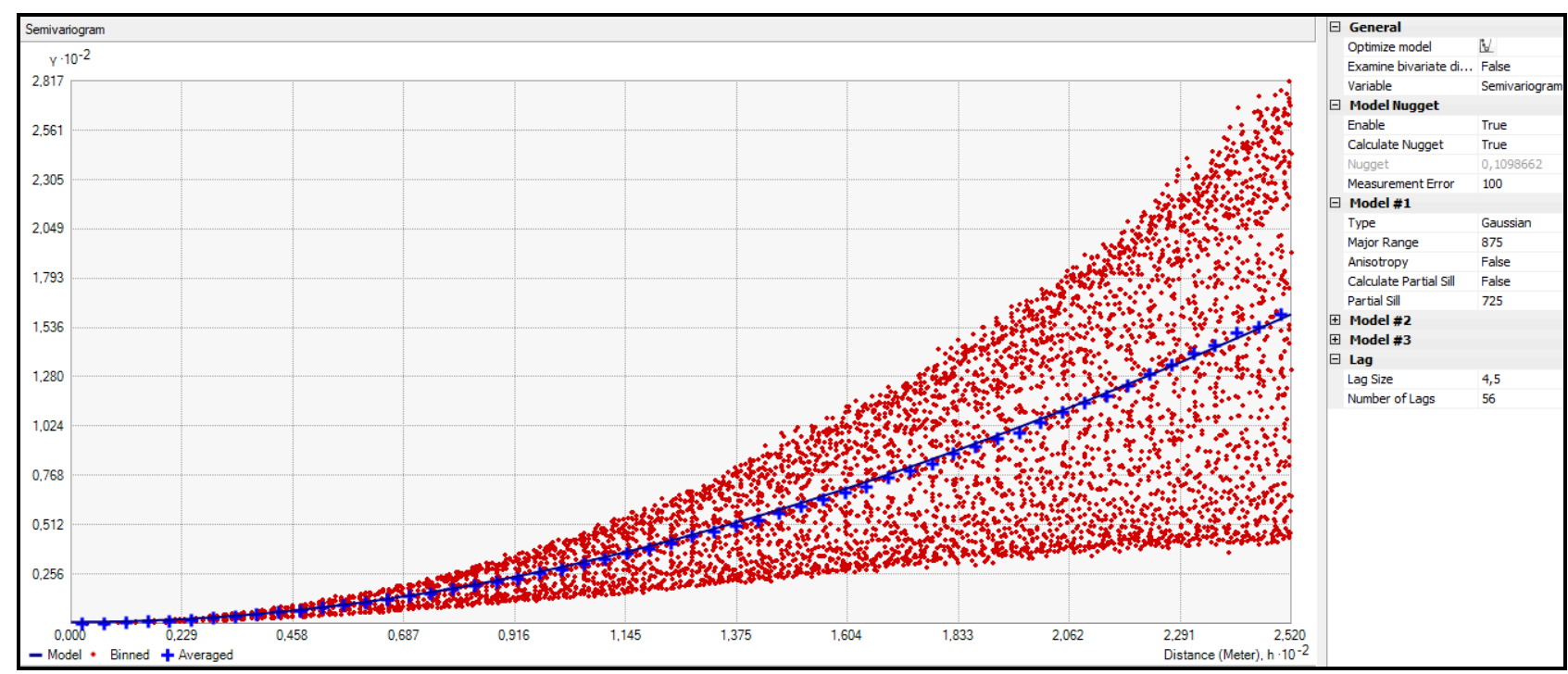

FONTE: Os autores (2019).

Revista Cerrados, Montes Claros/MG, v.17, n. 2, p. 247-264, jul./dez.-2019. 
PAZ, O. L. S.; SAMPAIO, T. V. M.

Geração de modelo digital do terreno e extração de parâmetros geomorfométricos a partir de dados coletados por aeronaves remotamente pilotadas

Os parâmetros geomorfométricos extraídos do MDT foram: declividade, orientação de vertente, plano e perfil de curvatura, acumulação de fluxo e índice topográfico de umidade (ITU). Os parâmetros foram calculados por análise de vizinhança, sendo definida uma janela móvel 3x3 pixels em estrutura grid.

A declividade foi calculada a partir das variáveis direcionais de Horn (1981), executada no ArcGIS 10.1 na ferramenta slope. O plano e perfil de curvatura foram calculados a partir das variáveis direcionais de Zevenbergen e Thorne (1987), pela ferramenta curvature no ArcGIS 10.1.

A acumulação de fluxo foi calculada pelo algoritmo D8 na ferramenta FlowDirection do ArcGIS 10.1. O Índice topográfico de umidade (ITU) representa área de saturação superficial de acordo com a topografia. Foi gerado calculando a acumulação de fluxo dividido pela tangente da declividade (MOORE et al., 1993). A fórmula para cálculo do ITU é apresentada abaixo.

$$
\operatorname{ITU}=\ln \left(\frac{A_{\mathrm{C}}}{\tan \beta}\right)
$$

Onde: $L n$ : logaritmo natural / $A_{c \text { : }}$ área de contribuição (acumulação de fluxo) / $\tan \beta$ : tangente da declividade mensurada em graus

Foram gerados perfis longitudinais considerando o divisor de águas, canal de drenagem e exutório da bacia. Foi utilizado a ferramenta interpolate shape e em seguida a ferramenta profile graph, ambos do ArcGIS 10.1. Na etapa final, foi analisado as quebras do relevo ou knickpoint pela ferramenta Knickpoint Finder (SALAMUNI et al., 2013). Os parâmetros da ferramenta foram fixados em 10.000 para escala de trabalho e 10 para equidistância em metros. Para comparar com o resultado do MDT RPA, a ferramenta foi aplicada no MDT do projeto Shuttle Radar Topography Mission - SRTM X, com 30 metros de resolução espacial, adquirido no endereço eletrônico da United States Geological Survey (USGS).

\section{RESULTADOS E DISCUSSÃO}

Foram coletadas pela RPA 105 imagens. O ortomosaico e o MDT gerados são apresentados na Erro! Fonte de referência não encontrada.. A nuvem de pontos gerada no Revista Cerrados, Montes Claros/MG, v.17, n. 2, p. 247-264, jul./dez.-2019. 
PAZ, O. L. S.; SAMPAIO, T. V. M.

Geração de modelo digital do terreno e extração de parâmetros geomorfométricos a partir de dados coletados por aeronaves remotamente pilotadas

Agisoft PhotoScan 1.2.4 apresentou aproximadamente 21 milhões de pontos. Após a filtragem no Agisoft PhotoScan, a nuvem de pontos apresentou aproximadamente 18 milhões de pontos. No ArcGIS 10.1 foram selecionados 30 mil pontos, pois acima deste valor o pacote geoestatístico apresentou instabilidade, resultando em falhas e fechamento do software.

Figura 4: Ortomosaico e MDT gerados.

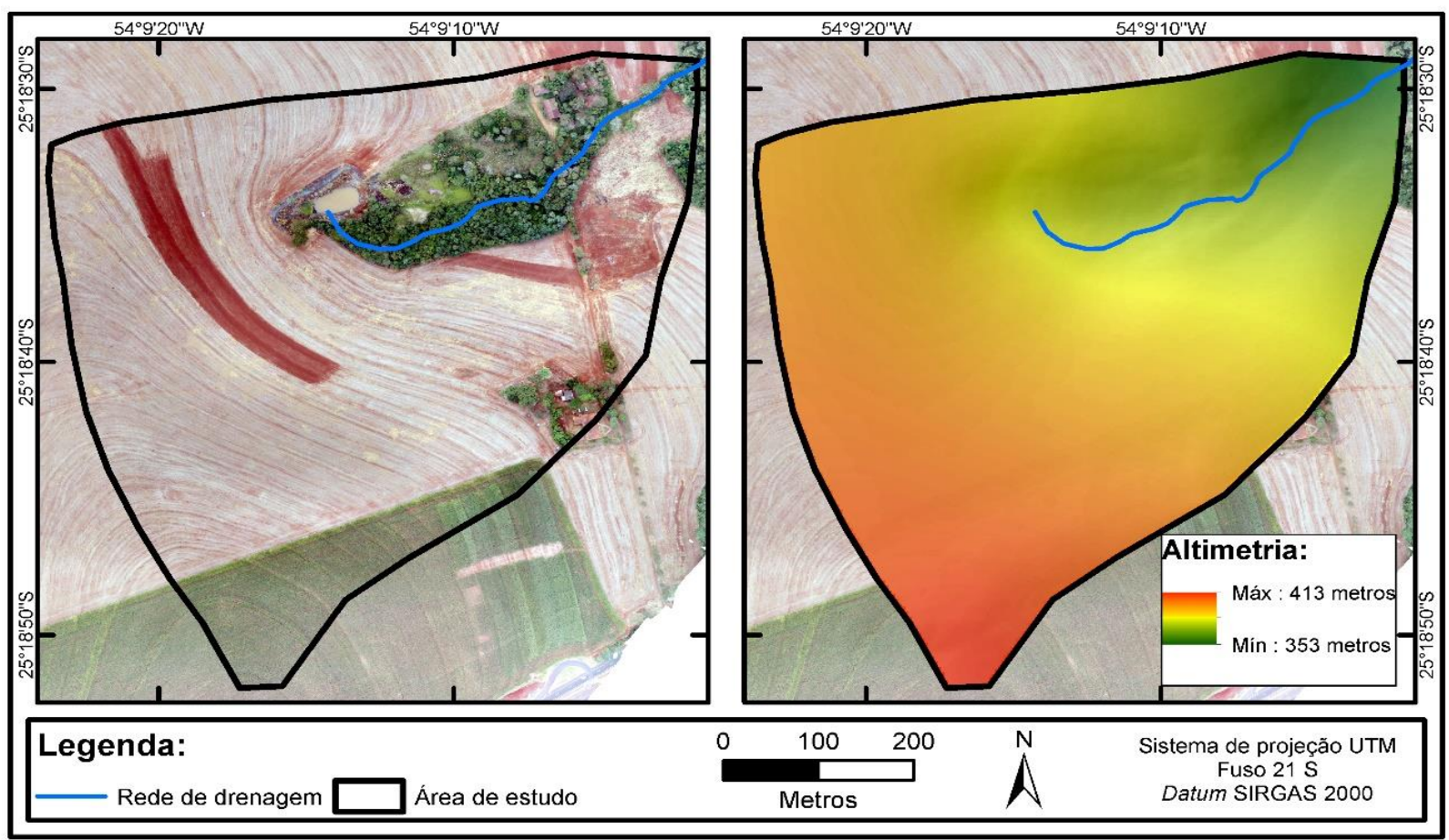

FONTE: Os autores (2019).

Os 30 mil pontos foram analisados com o ortomosaico visando retirar pontos de elementos sob o terreno. Após este procedimento e a análise do gráfico boxplot e dos polígonos de Voronoi, a nuvem de pontos final apresentou 28.982 pontos. Este valor representa aproximadamente $0,14 \%$ do tamanho da nuvem de pontos original gerada pelo Agisoft PhotoScan.

Não foram utilizados pontos de controle de campo para geração do ortomosaico e do MDT. No entanto, para uma noção da qualidade dos produtos gerados, podem ser feitas inferências de acordo com a literatura. O estudo realizado por Sopchaki et al. (2018) constatou que ortomosaicos gerados por dados coletados de RPA sem ponto de controle apresentaram enquadramento na classe A - 1:5.000 (DSG, 2016). Estudo semelhante foi 
PAZ, O. L. S.; SAMPAIO, T. V. M.

Geração de modelo digital do terreno e extração de parâmetros geomorfométricos a partir de dados coletados por aeronaves remotamente pilotadas

realizado por Hung et al. (2018) com foco no MDT. Os autores concluíram que o MDT gerado sem pontos de controle se enquadrava na classe A - 1:5.000 (DSG, 2016). Assim, espera-se que o ortomosaico e MDT gerado para a área de estudo também apresentem estes enquadramentos.

Os parâmetros geomorfométricos extraídos são apresentados na Erro! Fonte de referência não encontrada.. A altimetria da área de estudo apresenta amplitude de 60 metros, variando de 353 a 413 metros. A variação dos demais parâmetros geomorfométricos será descrita a seguir tendo como ponto de partida as vertentes encontradas.

Figura 5: Parâmetros geomorfométricos extraídos do MDT RPA.

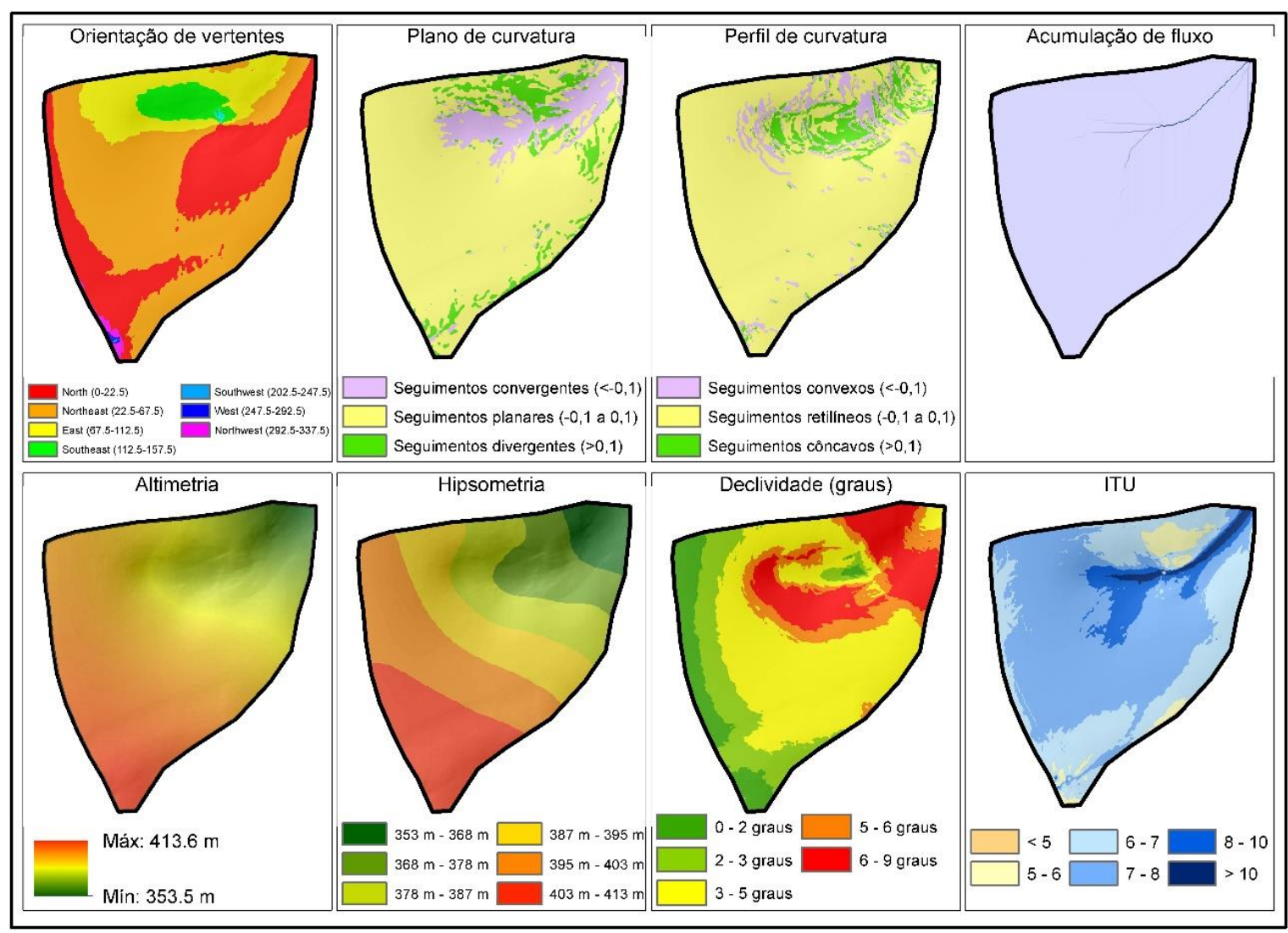

FONTE: Os autores (2019).

$\mathrm{Na}$ área predominam vertentes orientadas para norte-nordeste, tendo formato planar-retilíneo (Erro! Fonte de referência não encontrada. - A). Estas características estão relacionadas aos demais parâmetros geomorfométricos. A declividade nestas áreas vai até 5 graus. Analisando a forma de vertente encontrada nesta área pode-se afirmar que os fluxos 


\section{PAZ, O. L. S.; SAMPAIO, T. V. M.}

Geração de modelo digital do terreno e extração de parâmetros geomorfométricos a partir de dados coletados por aeronaves remotamente pilotadas

advindos de precipitação seguem para o vale da bacia, não ocorrendo acumulação, estando esta característica relacionada aos baixos valores do ITU nesta área.

Figura 6: A - Vertente orientada para norte-nordeste, tendo formato planar-retilínea. B -

Vertente com formato convergente-côncava.
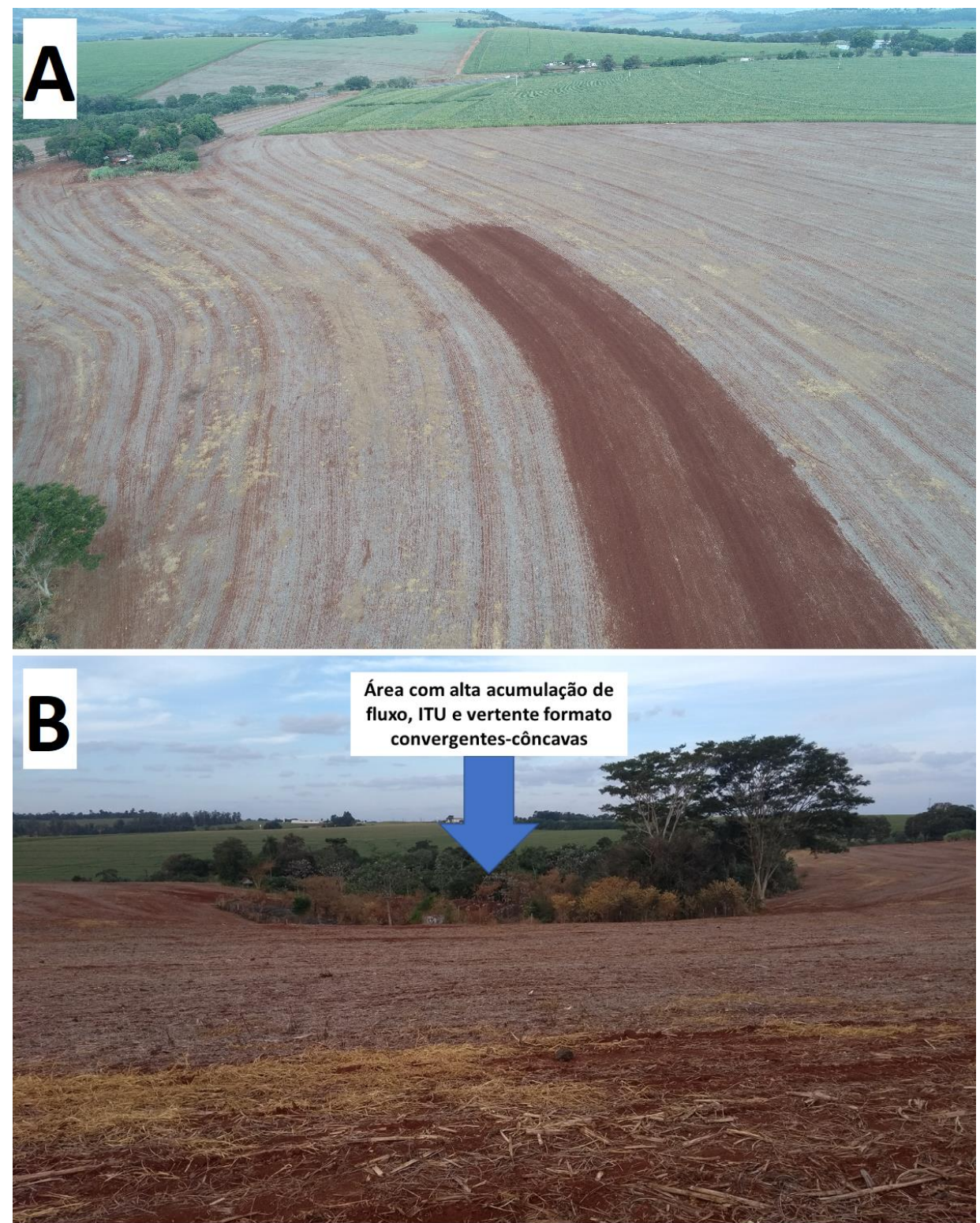

FONTE: Os autores (2019).

As áreas com maior declividade foram encontradas no vale do rio de primeira ordem, variando de 5 a 9 graus. Consequentemente, é neste vale que se encontram os maiores valores de acumulação de fluxo, do ITU e seguimentos de vertentes convergentes-côncavas. A 
PAZ, O. L. S.; SAMPAIO, T. V. M.

Geração de modelo digital do terreno e extração de parâmetros geomorfométricos a partir de dados coletados por aeronaves remotamente pilotadas

Erro! Fonte de referência não encontrada. - B apresenta fotografia dessa porção da bacia de primeira ordem.

A FIGURA 7 ilustra a interação entre as duas formas de vertentes descritas anteriormente. Os fluxos das vertentes planares-retilíneas seguem a porção do vale (convergentes-côncavas), onde está o rio de primeira ordem. O círculo vermelho na Erro! Fonte de referência não encontrada. representa uma quebra no relevo observada em campo. Essa ruptura observada se encaixa no conceito de knickpoints: quebras ou rupturas de declive, que criam anomalias de relevo no perfil longitudinal (CROSBY; WHIPPLE, 2006).

Figura 7: Interação da área com vertentes planar-retilínea (em amarelo) com vertentes convergentes-côncavas (azul). O círculo vermelho representa a ruptura do relevo observado em campo. Imagem registrada pela RPA a 60 metros de altura.

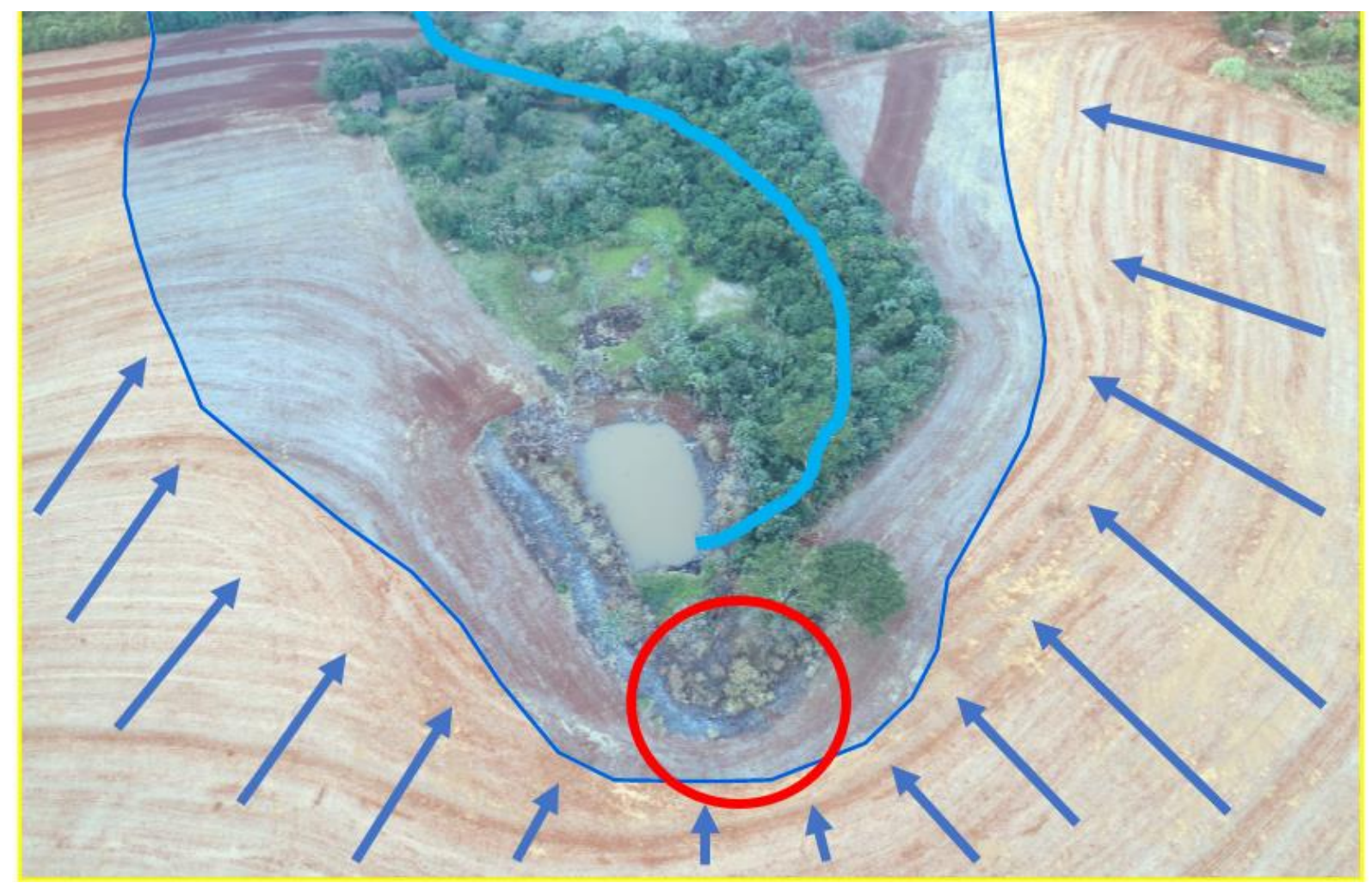

FONTE: Os autores (2019).

A parte central do perfil longitudinal do divisor até o exutório mostra a quebra no relevo observado em campo (FIGURA 8). Quando aplicado a ferramenta Knickpoint Finder (SALAMUNI et al., 2013) verifica-se a presença de 2 knickpoints. O mais a montante 
PAZ, O. L. S.; SAMPAIO, T. V. M.

Geração de modelo digital do terreno e extração de parâmetros geomorfométricos a partir de dados coletados por aeronaves remotamente pilotadas

corresponde ao observado em campo. Este apresentou índice de Relação DeclividadeExtensão (RDEs) de 10,3, enquanto o knickpoint a jusante apresentou RDEs de 0,77.

Figura 8: Perfil longitudinal e knickpoint identificados no MDP RPA.
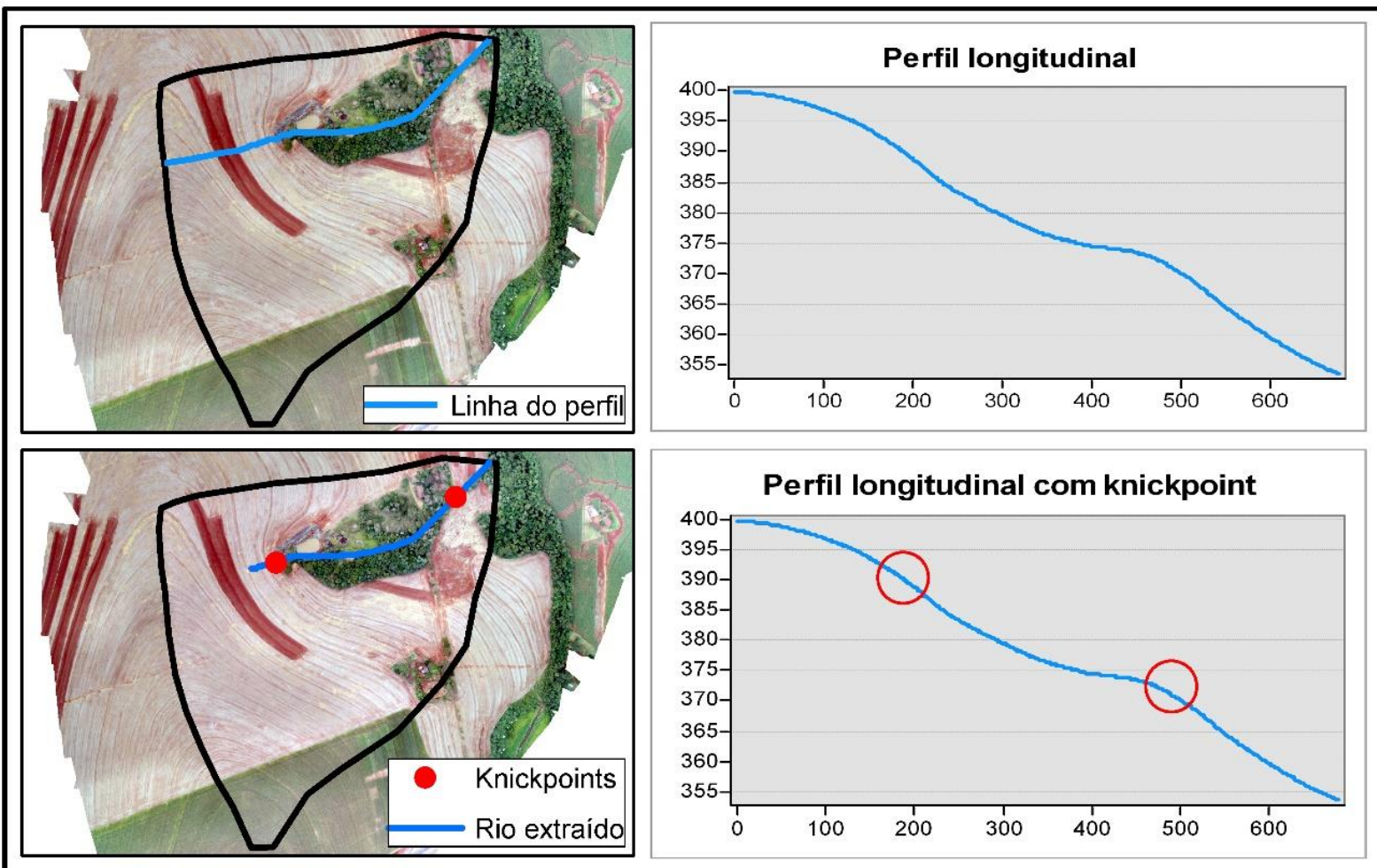

FONTE: Os autores (2019).

Quando se analisa o resultado da aplicação no MDT SRTM X nota-se que a identificação de 1 knickpoint próximo ao exutório da bacia, semelhante ao encontrado na aplicação da ferramenta Knickpoint Finder no MDT RPA (FIGURA 9). No entanto, o knickpoint observado em campo não foi identificado no MDT SRTM X, o que indica que o MDT RPA possibilita maior detalhamento na análise do relevo da bacia. 
PAZ, O. L. S.; SAMPAIO, T. V. M.

Geração de modelo digital do terreno e extração de parâmetros geomorfométricos a partir de dados coletados por aeronaves remotamente pilotadas

Figura 9: Perfil longitudinal e knickpoint identificados no MDP RPA.

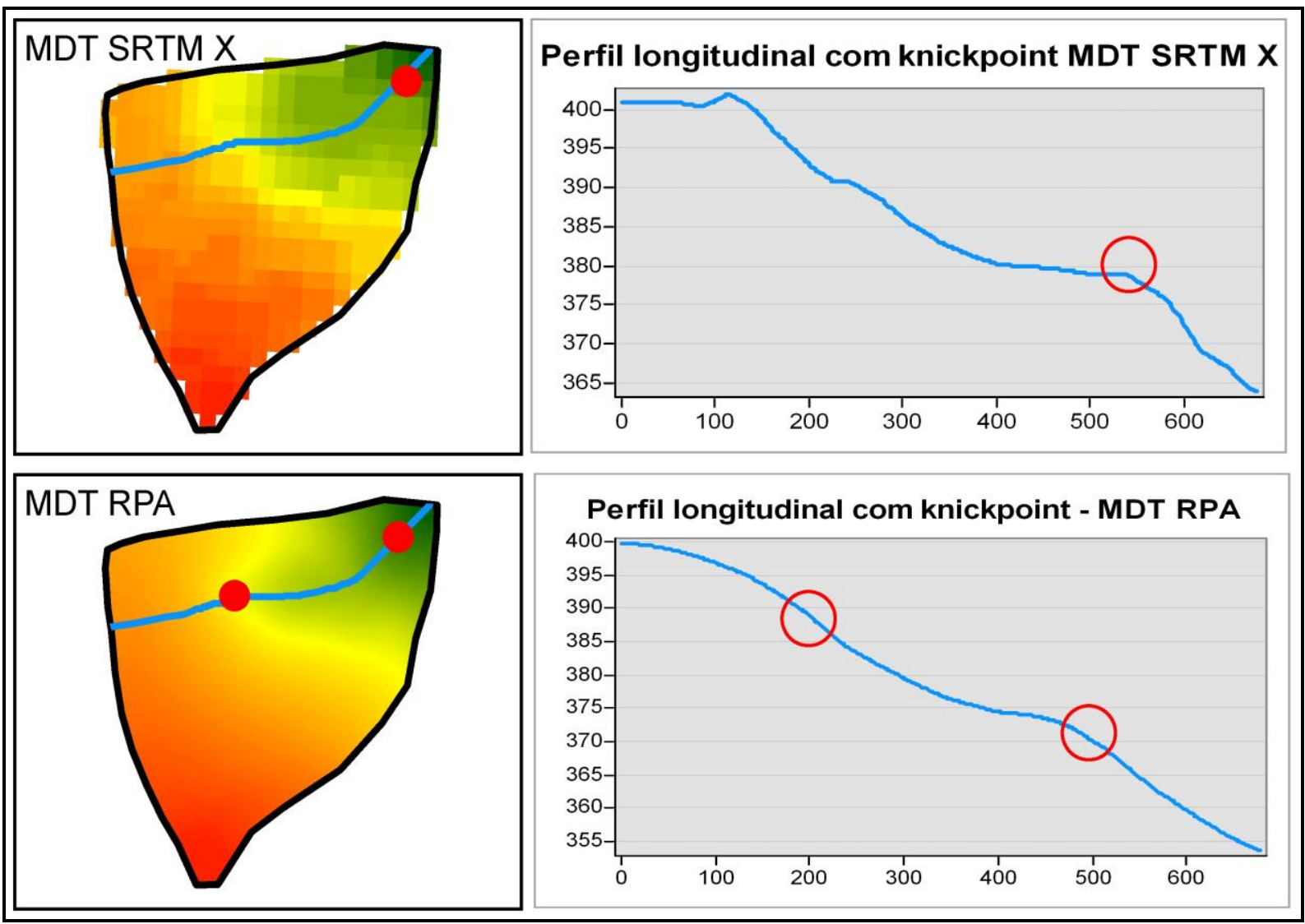

FONTE: Os autores (2019).

Em síntese, a aplicação de RPA na captura de imagens em alta resolução espacial permite maior detalhamento no MDT derivado. A partir deste, foi possível identificar feições de detalhe verificadas em campo por meio dos parâmetros geomorfométricos extraídos. No entanto, apesar de existirem trabalhos sobre qualidade altimétrica do MDT gerado, ainda não é claro a qualidade dos parâmetros geomorfométricos extraídos destes MDT, sendo necessários estudos mais aprofundados.

Também vale salientar que a área de estudo adotada nesta pesquisa apresenta solo exposto em sua maior parte, o que facilitou o processo de filtragem da nuvem de pontos para interpolação/geração do MDT. Áreas com a presença de vegetação em diversos estratos e/ou densidades ou edificações podem apresentar maior dificuldade na filtragem da nuvem pontos, resultando em nuvem de pontos de diferentes distribuições e densidades, o que consequentemente pode afetar o MDT gerado e parâmetros geomorfométricos extraídos. 
PAZ, O. L. S.; SAMPAIO, T. V. M.

Geração de modelo digital do terreno e extração de parâmetros geomorfométricos a partir de dados coletados por aeronaves remotamente pilotadas

\section{CONSIDERAÇÕES FINAIS}

Com imagens coletadas por RPA obteve-se um MDT e ortomosaico em escala de detalhe para a área de estudo. A partir do MDT foram extraídos parâmetros que contribuíram para caracterização geomorfométrica em detalhe da área. A bacia de primeira ordem localizada a oeste de Medianeira apresentou vertentes planar-retilíneas com declividade de até 5 graus e baixos valores de ITU. Nas áreas do vale do rio de primeira ordem, observa-se vertentes convergentes-côncavas marcadas por declividades entre 5 a 9 graus, associadas a altos valores de ITU.

O MDT gerado pelo aerolevantamento com RPA permitiu a identificação de um knickpoint observado em campo. Este não foi identificado quando utilizado o MDT SRTM X, o que possibilita afirmar que o MDT gerado permite análise em detalhe do relevo da bacia de primeira ordem. Assim, nota-se o potencial de dados coletados por RPA para caracterização em detalhe do relevo. Este potencial pode ser aplicação em estudos de caracterização ambiental, projeto de obras, mapeamento preditivo de solos, planejamento de plantio e colheita, entre outros.

\section{AGRADECIMENTOS}

Os autores agradecem o apoio financeiro da Coordenação de Aperfeiçoamento de Pessoal de Nível Superior - Brasil (CAPES) pela concessão da bolsa de pesquisa ao primeiro autor. Os autores agradecem também ao Conselho Nacional de Desenvolvimento Científico e Tecnológico $(\mathrm{CNPq})$ pela verba disponibilizada no projeto de pesquisa "Mapeamento da rede de drenagem: avaliação da acurácia da representação cartográfica da rede de drenagem no estado do Paraná" no processo n. ${ }^{\circ}$ 421960/2016-7.

\section{REFERÊNCIAS}

ARRUDA, G. P. de; DEMATTÊ, J. A. M.; SILVA CHAGAS, C. Mapeamento digital de solos por redes neurais artificiais com base na relação solo-paisagem. Revista Brasileira de Ciência do Solo, v. 37, n. 2, 2013. Disponível em: < http://www.scielo.br/scielo.php?pid=S0100-

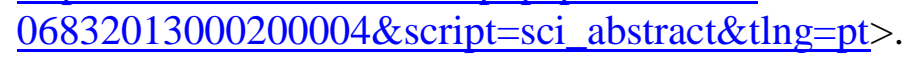

ARUN, P. V. A comparative analysis of different DEM interpolation methods. The Egyptian Journal of Remote Sensing and Space Science, v. 16, n. 2, p. 133-139, 2013. 
PAZ, O. L. S.; SAMPAIO, T. V. M.

Geração de modelo digital do terreno e extração de parâmetros geomorfométricos a partir de dados coletados por aeronaves remotamente pilotadas

CHORLEY, R. J. Climate and Morphometry. The Journal of Geology, v. 65, n. 6, p. 628638, 1957. Disponível em: <https://doi.org/10.1086/626468>.

CROSBY, B. T.; WHIPPLE, K. X. Knickpoint initiation and distribution within fluvial networks: 236 waterfalls in the Waipaoa River, North Island, New Zealand. Geomorphology, v. 82, n. 1-2, p. 16-38, 2006.

DSG - DIRETORIA DO SERVIÇO GEOGRÁFICO. Especificação técnica para a aquisição de dados geoespaciais vetoriais (ET-ADGV). Ministério da Defesa, Exército Brasileiro, Departamento de Ciência e Tecnologia. Brasília-DF, $2^{\mathrm{a}}$ edição, v. 2, 2016.

GUJJAR, P. et al. The MIR Flickr Retrieval Evaluation Proposal Based on User Tags and Textual Passwords. International Journal of Advanced Engineering, Management and Science, n. 4, p. 343-349, 2017.

HUNG, M. N. W. B. et al. Levantamento com veículo aéreo não tripulado para geração de modelo digital do terreno em bacia experimental com vegetação florestal esparsa. Raega-O Espaço Geográfico em Análise, v. 43, p. 215-231, 2018.

MARION, F. A.; MELLO FILHO, J. A.; SILVA, J. L. S. Análise da vulnerabilidade natural das águas subterrâneas por geoprocessamento no campus da UFSM-RS. Terr@ Plural, v. 4, n. 1, p. 65-76, 2010. Disponível em:

<http://www.revistas2.uepg.br/index.php/tp/article/view/1132>.

MOHAMMAD, S. et al. The Understanding of Exchangeable Image File ( Exif ) Metadata of Images : Towards Disseminating the Awareness to the Society. In: 3th International Conference on Information Technology \& Society, Anais...2017.

MOORE, I. D. et al. Soil attribute prediction using terrain analysis. Soil Science Society of America Journal, v. 57, n. 2, p. 443-452, 1993.

OLAYA, V. Basic land-surface parameters. Developments in Soil Science, v. 33, p. 141169, 2009. Disponível em:

<https://www.sciencedirect.com/science/article/pii/S0166248108000068>.

PIKE, R. J. Geomorphometry - diversity in quantitative surface analysisProgress in Physical Geography, 2000. .

RAAFLAUB, L. D.; COLLINS, M. J. The effect of error in gridded digital elevation models on the estimation of topographic parameters. Environmental Modelling \& Software, v. 21, n. 5, p. 710-732, 2006.

REICHEL, H. R.; SAMPAIO, T. V. M. Geração de modelo digital do terreno (MDT) a partir da integração de métodos geoestatísticos e dados obtidos com uso de aeronaves remotamente pilotadas (RPAS). In: Anais do XII Simposío Nacional de Geomorfologia, Crato. Anais...

Crato: 2018. Disponível em: <http://www.sinageo.org.br/2018/trabalhos/9/9-418-2071.html>. 
PAZ, O. L. S.; SAMPAIO, T. V. M.

Geração de modelo digital do terreno e extração de parâmetros geomorfométricos a partir de dados coletados por aeronaves remotamente pilotadas

SAHA, A. K.; GUPTA, R. P.; ARORA, M. K. GIS-based landslide hazard zonation in the Bhagirathi (Ganga) valley, Himalayas. International journal of remote sensing, v. 23, n. 2, p. 357-369, 2002. Disponível em:

<https://www.tandfonline.com/doi/abs/10.1080/01431160010014260>.

SALAMUNI, E. et al. Knickpoint Finder: ferramenta para a busca de geossítios de relevante interesse para o geoturismo. Boletim Paranaense de Geociências, v. 70, 2013.

SILVA, J. M. F.; OKA-FIORI, C.; SILVEIRA, C. T. Geomorfometria: uma Análise de Conceitos, Métodos e Aplicações em Geografia Física. Revista Brasileira de Geografia Física, v. 10, n. 2, p. 558-583, 2017. Disponível em:

<https://periodicos.ufpe.br/revistas/rbgfe/article/view/234008/27453>.

SILVEIRA, R. M. P. et al. ANÁLISE DIGITAL DO RELEVO EMPREGADA NO MAPEAMENTO DE UNIDADES GEOMORFOLÓGICAS. Geografar, v. 7, p. 43-68, 2012. Disponível em: <https://revistas.ufpr.br/geografar/article/view/26709>.

SILVEIRA, R. M. P.; DA SILVEIRA, C. T. Análise digital do relevo aplicada à cartografia geomorfológica da porção central da Serra do Mar Paranaense. Revista Brasileira de Geomorfologia, v. 17, n. 4, 2016.

SOPCHAKI, C. H. et al. VERIFICAÇÃO DA QUALIDADE DE ORTOMOSAICOS PRODUZIDOS A PARTIR DE IMAGENS OBTIDAS COM AERONAVE REMOTAMENTE PILOTADA SEM O USO DE PONTOS DE APOIO. Raega - O Espaço Geográfico em Análise, v. 43, p. 200, 2018.

SOPCHAKI, C. H.; SAMPAIO, T. V. M. Airborne Laser Scanner : principles of operation, recent uses in Brazil and regulatory issue from laws and parameters in Brazil and in the USA. Journal of Hyperspectral Remote Sensing, v. 6, n. 7, p. 338-353, 2016. Disponível em: <http://www.revista.ufpe.br/jhrs/index.php/revista/article/view/126/pdf>.

TACHIKAWA, T. et al. Characteristics of ASTER GDEM version 2. In: International Geoscience and Remote Sensing Symposium (IGARSS), Anais...2011. Disponível em: $<$ https://ieeexplore.ieee.org/document/6050017>.

WESTOBY, M. J. et al. 'Structure-from-Motion'photogrammetry: A low-cost, effective tool for geoscience applications. Geomorphology, v. 179, p. 300-314, 2012. Disponível em: <https://www.sciencedirect.com/science/article/pii/S0169555X12004217>. 


\section{Autores}

Otacílio Lopes de Souza da Paz - Possui Graduação e Mestrado em Geografia pela Universidade Federal do Paraná (UFPR). Atualmente é doutorando pelo Programa de PósGraduação em Geografia da Universidade Federal do Paraná (UFPR).

Tony Vinicius Moreira Sampaio - Possui Graduação em Geografia pela Universidade Federal do Espírito Santo (UFES), Mestrado e Doutorado em Geografia, ambos pela Universidade Federal de Minas Gerais (UFMG). Atualmente é Professor do Programa de PósGraduação em Geografia da Universidade Federal do Paraná (UFPR).

Artigo recebido em: 06 de setembro de 2019

Artigo aceito em: 05 de dezembro de 2019

Artigo publicado em: 20 de dezembro de 2019 